\title{
The nature of the X-ray halo of the plerion G21.5-0.9 unveiled by XMM-Newton and Chandra
}

\author{
F. Bocchino ${ }^{1}$, E. van der Swaluw ${ }^{2,3}$, R. Chevalier ${ }^{4}$, and R. Bandiera ${ }^{5}$
}

\author{
1 INAF - Osservatorio Astronomico di Palermo, Piazza del Parlamento 1, 90134 Palermo, Italy \\ e-mail: bocchino@astropa.unipa.it \\ 2 FOM - Institute for Plasma Physics Rijnhuizen, PO Box 1207, 3430 BE Nieuwegein, The Netherlands \\ 3 School of Physics and Astronomy, The University of Leeds, Woodhouse Lane, Leeds LS2 9JT, UK \\ 4 Department of Astronomy, University of Virginia, PO Box 3818, Charlottesville, VA 22903, USA \\ 5 INAF - Osservatorio Astrofisico di Arcetri, Largo E. Fermi 5, 50125 Firenze, Italy
}

Received 11 February 2005 / Accepted 15 July 2005

\section{ABSTRACT}

\begin{abstract}
The nature of the radio-quiet X-ray halo around the plerionic supernova remnant G21.5-0.9 is under debate. On the basis of spatial and spectral analysis of a large Chandra and XMM-Newton dataset of this source, we have developed a self-consistent scenario which explains all the observational features. We found that the halo is composed by diffuse extended emission due to dust scattering of X-rays from the plerion, by a bright limb which traces particle acceleration in the fast forward shock of the remnant, and by a bright spot (the "North Spur") which may be a knot of ejecta in adiabatic expansion. By applying a model of interaction between the PWN, the remnant and the supernova environment, we argue that G21.5-0.9 progenitor may be of Type IIP or Ib/Ic, and that the remnant may be young (200-1000 yr).
\end{abstract}

Key words. ISM: supernova remnants - ISM: dust, extinction - X-rays: ISM - X-rays: individuals: G21.5-0.9 stars: supernovae: general - radiation mechanisms: non-thermal

\section{Introduction}

The plerionic supernova remnant (SNR) G21.5-0.9 has been extensively studied in radio (see e.g. Becker \& Kundu 1976; Becker \& Szymkowiak 1981; Fürst et al. 1988; Kassim 1992; Bock et al. 2001, and references therein) and in the X-ray band (e.g. Davelaar et al. 1986; Asaoka \& Koyama 1990; Slane et al. 2000; Warwick et al. 2001; Safi-Harb et al. 2001). In spite of several efforts, its pulsar remains undetected (Biggs \& Lyne 1996; La Palombara \& Mereghetti 2002). Woltjer et al. (1997) include it among the non-Crab like class of plerions, because of its low frequency spectral break, for which a non standard evolutionary path of the pulsar output must be invoked. However, Bock et al. (2001) and Bandiera et al. (2001) pointed out that new observations, at 94 and $230 \mathrm{GHz}$ respectively, suggest a spectral break above $100 \mathrm{GHz}$, much higher than previously thought.

A set of new and detailed observations in the X-ray band performed with XMM-Newton and Chandra have apparently raised new and interesting questions about the nature of this object, and in particular on the extended and diffuse X-ray halo which seems to surround this plerion. Slane et al. (2000) seem to have been the first to detect X-ray emission extending beyond the boundary of the radio plerion. The short Chandra calibration observation they used prevented a detailed study of the halo, and it was not recognized if the emission was thermal or non-thermal. Due to the lack of further data, Slane et al. (2000) suggested that the halo may represent the shell formed by the interaction of the main blast wave with the surrounding medium. They examined archival VLA radio data and concluded that the upper limit to the $1 \mathrm{GHz}$ surface brightness $(1 \sigma)$ is $4 \times 10^{-21} \mathrm{~W} \mathrm{~m}^{-2} \mathrm{~Hz}^{-1} \mathrm{sr}^{-1}$. Warwick et al. (2001) used the XMM-Newton calibration observation of G21.5-0.9 we also use, and established the non-thermal nature of the $\mathrm{X}$-ray emission of the halo. The lack of line emission in the integrated spectra of the halo pointed toward a very small ionization time $\left(\sim 3 \times 10^{8} \mathrm{~cm}^{-3} \mathrm{~s}\right)$, if non-equilibrium of ionization were used in the fit. Warwick et al. (2001) also noted that the size of the X-ray halo exceeds that of the radio PWN (pulsar wind nebula) by a factor of 4 , a feature which is not expected and indeed not observed in any other PWN. They also detected a bright spot (named "North Spur") and some filaments in the halo. Safi-Harb et al. (2001) confirmed the Warwick et al. (2001) findings and considered the halo as an extension of the plerion nebula, but they also pointed out that the observed morphology is puzzling and cannot be explained by diffusion models. The X-ray morphology was also briefly discussed by Bock et al. (2001), who confirmed the previous upper limit on $\Sigma_{1 \mathrm{GHz}}$, 
and who suggested multiple events or injection epochs, noting, however, that the new radio data do not address the question of why the morphology is so peculiar. Bandiera \& Bocchino (2004) proposed that the X-ray halo is due to photons scattered by ISM dust along the line of sight, and showed that this model is broadly compatible with the observed absorption and radial surface brightness profiles. However, their fit to the profiles showed residuals due the presence of the (then undetected) weak features in the halo, and an error in the profile normalization led to the conclusion that an intrinsic halo was required to fit the data in the 5-8 keV. Finally, Bocchino (2005) has reported the detection of a weak thermal component in the X-ray spectrum of the "North Spur" as seen by both Chandra and $X M M-N e w t o n$. While the metal abundances seem to indicate that the North Spur is an ejecta knot, the measured X-ray temperature is much lower than the expected ejecta temperature behind a reverse shock.

It is clear that the nature of the X-ray halo of G21.5-0.9 is still poorly known. Most of the studies have been focused on the central plerion and there has been no systematic study of the halo itself. As a consequence, the three possible models for the X-ray halo introduced above, namely the non-thermal shell, the extension of the plerion and the dust-scattering of $\mathrm{X}$-rays from the center, have not been properly investigated. In this paper, using new X-ray datasets that have been accumulated by Chandra and XMM-Newton, we propose a possible scenario which explains the halo as a non-thermal shell superposed on a dust-scattering profile. We will show that the proposed interpretation nicely fits all the observational evidence and is in agreement with evolutionary models of young PWN and SNR. In particular, in Sect. 2 we present the dataset we have used, in Sect. 3 we discuss the morphology of the X-ray halo, in Sect. 4 we perform spatially resolved spectroscopy of the halo and its features, in Sect. 5 we introduce the dust scattering model which explains part of the halo emission, while in Sect. 6 we compare our findings with evolutionary models of young PWN-SNRs.

\section{Observations}

G21.5-0.9 was observed as part of the Calibration and Performance Verification phase of the XMM-Newton satellite (Jansen et al. 2001). In particular, the remnant was observed both on-axis and off-axis (for the list of G21.5-0.9 observations, see Table 1). In this work, we have used the array of $\mathrm{PN}$ CCDs (Strüder et al. 2001) and the two arrays of MOS CCDs (Turner et al. 2001) at the focus of three X-ray mirrors of XMM-Newton (Gondoin et al. 1998). The nominal bandwidth, angular resolution and energy resolution of the instruments are $0.1-15 \mathrm{keV}, 15^{\prime \prime} F W H M$ and $E / \Delta E \sim 10$, respectively. The original event file was screened to eliminate the contribution of soft protons, from both flares and quiescent emission, using the recipe given by De Luca \& Molendi (2004). Since the observation numbers 3 and 4 were found to be affected by unusually large quiescent emission, they were discarded in the analysis of the dimmest sources (middle and outer halo, see below). All the analysis of XMM data was performed with the software SAS v6.0.
Table 1. Cal/PV XMM-Newton observations of G21.5-0.9.

\begin{tabular}{lcccc}
\hline \hline Obs. & Pointing & \multicolumn{2}{c}{$T_{\text {in }} / T_{\text {out }}(\mathrm{ks})$} & Date \\
& location & PN & MOS & \\
\hline OnAxis & on G21.5-0.9 & $27 / 18$ & $30 / 25$ & 7 Apr. 2000 \\
OffAxis1 & $10^{\prime} \mathrm{S}$ & $28 / 26$ & $29 / 25$ & 9 Apr. 2000 \\
OffAxis2 & $10^{\prime} \mathrm{W}$ & $28 / 23$ & $29 / 27$ & 11 Apr. 2000 \\
OffAxis3 & $10^{\prime} \mathrm{N}$ & $29 / 06$ & $29 / 19$ & 15 Apr. 2000 \\
OffAxis4 & $10^{\prime} \mathrm{E}$ & $29 / 06$ & $29 / 20$ & 17 Apr. 2000 \\
\hline
\end{tabular}

${ }^{a}$ At the coordinates $18^{\mathrm{h}} 33^{\mathrm{m}} 32.6^{\mathrm{s}}$ and $-10^{\mathrm{d}} 33^{\mathrm{m}} 57^{\mathrm{s}}(\mathrm{J} 2000)$.

G21.5-0.9 was also observed as part of the calibration plan for the Chandra satellite (Weisskopf et al. 1996). There are $\sim 70$ observations available up to July 2004, among which we selected only the 21 observations for which G21.5-0.9 was located onto the S3 chip and at an off-axis angle less then 5 arcmin. The observation IDs are 0159, 1433, 1554, 1717, 1769, 1771, 1839, 2873, 3693, 4353, 5166, 1230, 1553, 1716, $1718,1770,1838,1840,3474,3700,4354$, and the total exposure time is $196.5 \mathrm{ks}$. These are the same observations used by Bocchino (2005). Our set includes the set used by Safi-Harb et al. (2001) plus the more recent observations that have been done (they used 6 observations for a total of $65 \mathrm{ks}$ ). Afterward, the data were screened for bad grades and for a clean status column. All the filtered datasets were merged together using the CIAO MERGE_ALL task.

\section{X-ray morphology of the halo}

In Fig. 1 we show the images of G21.5-0.9 as seen by the Chandra ACIS-S camera in two energy bands (0.2-2.0 keV and $2.0-10.0 \mathrm{keV}$, respectively). The figure shows the prominent X-ray halo around the bright plerion (the latter is marked by radio emission contours) and a compact bright feature in it at the location $18^{\mathrm{h}} 33^{\mathrm{m}} 32.9^{\mathrm{s}}$ and $-10^{\mathrm{d}} 32^{\mathrm{m}} 49^{\mathrm{s}}(\mathrm{J} 2000)$, the so called "North Spur" (NS hereafter), located at 80" from the center ( $\sim 1.9 \mathrm{pc}$ at $5 \mathrm{kpc}$ distance). A search for counterparts of the North Spur in other wavelengths performed both with catalog browsing (SIMBAD) and image retrieving (Skyview) has revealed no obvious counterparts within a radius of $20^{\prime \prime}$. The circular symmetry of the halo at $\mathrm{PA}=210^{\circ}-315^{\circ}$ is remarkable (here we adopt $\mathrm{PA}=0$ at $\mathrm{N}$, positive clockwise) and a bright limb is present. The limb is outlined by the white circle in Fig. 1, which has a radius of $138^{\prime \prime}(\sim 3.3$ pc at a distance of $5 \mathrm{kpc})$ and a center located $8.7^{\prime \prime}(0.21 \mathrm{pc})$ in the Southeast direction with respect to the peak of the PWN. At PA between $-45^{\circ}$ and $60^{\circ}$ we found the NS and bright diffuse emission apparently associated to it. This emission is in the form of weak arc-shaped filaments which surround the North Spur and connect it to both the plerion and the bright limb. In the remaining part of the halo (approximately from $\mathrm{PA}=60^{\circ}$ and $\mathrm{PA}=180^{\circ}$, no other features are detected, apart from the star SS397, no limb brightening is evident, and the halo declines more rapidly toward the background.

Surface brightness profiles of the halo of G21.5-0.9 have been derived using the XMM-Newton observations, and are shown in Fig. 2. All the profiles are computed using a weighted average of the PN, MOS1 and MOS2 data, where the weights 


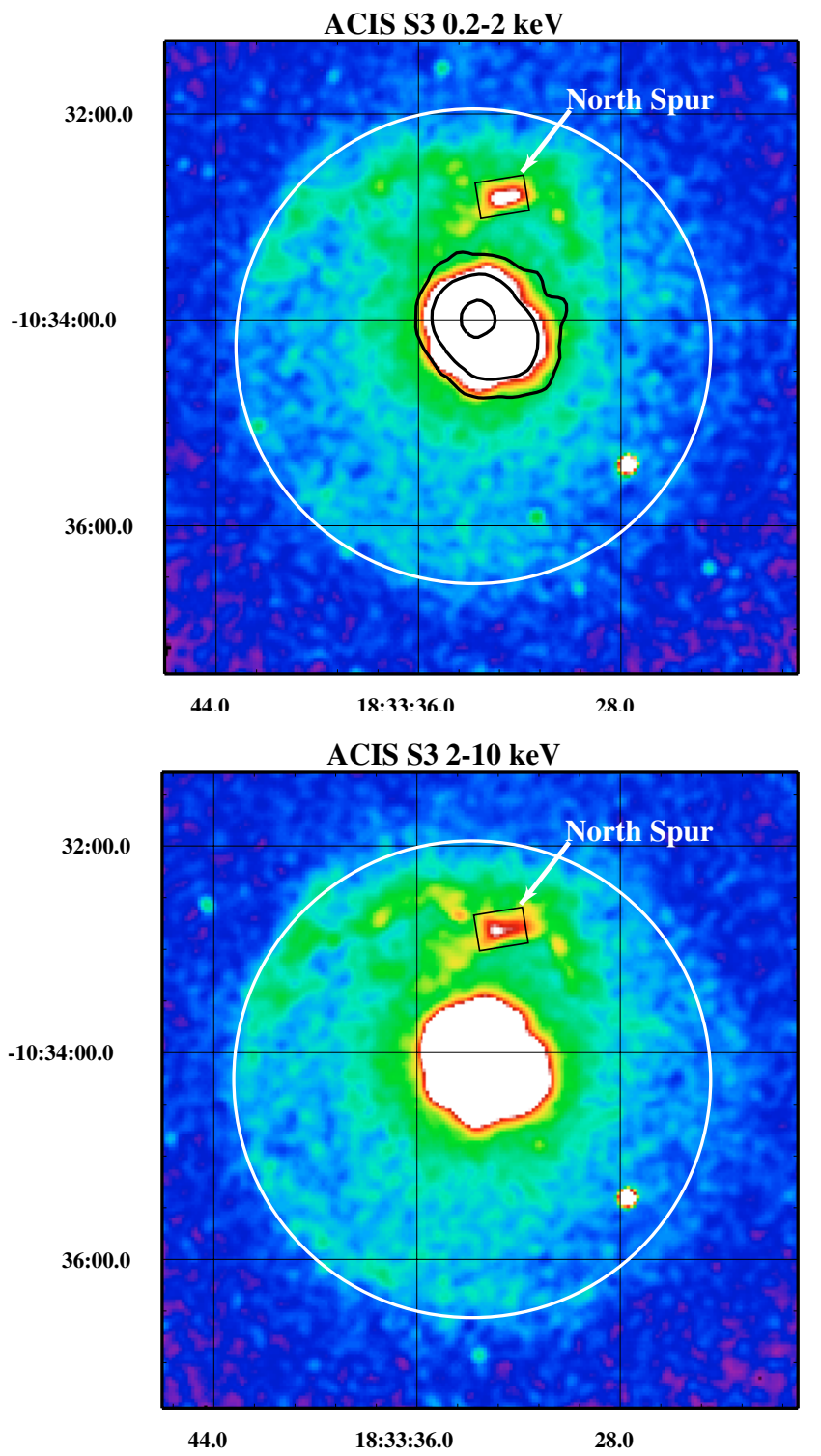

Fig. 1. Chandra ACIS-S images of G21.5-0.9 in the $0.2-2 \mathrm{keV}$ (top panel) and in the $2-10 \mathrm{keV}$ band (bottom panel). The color has been chosen to emphasize the weak halo emission. Black contours represent $22.3 \mathrm{GHz}$ contours of the large-scale diffuse emission of G21.5-0.9 at 10, 50 and $100 \mathrm{mJy} /$ beam ( 8 arcsec HEBW) from Fürst et al. (1988). The position of the North Spur is indicated. The white circle fits the outer limb brightened emission of the X-ray halo, it has a radius of $138 \operatorname{arcsec}(\sim 3.3 \mathrm{pc}$ at $5 \mathrm{kpc})$ and a center at $8.7^{\prime \prime}$ from the peak of the PWN.

have been derived in each band assuming a non-thermal spectrum with a photon index of 2 and an absorption of $2 \times$ $10^{22} \mathrm{~cm}^{-2}$, which is a good estimate of the halo spectrum. In order to study the profile of the "pure" halo, that is without the discrete feature, we have selected only data in the PA range $60^{\circ}-160^{\circ}$. All the profiles have been background subtracted using a local background (collected in a large annulus between 280 and 320 arcsec) and vignetting corrected. Figure 2 shows that G21.5-0.9 has a sharp change in its X-ray profiles around $50^{\prime \prime}(\sim 1.2 \mathrm{pc})$ from the center, where the plerion profile (which is narrower than the radio counterpart, as expected) suddenly flattens and continues out to $\sim 250^{\prime \prime}$.

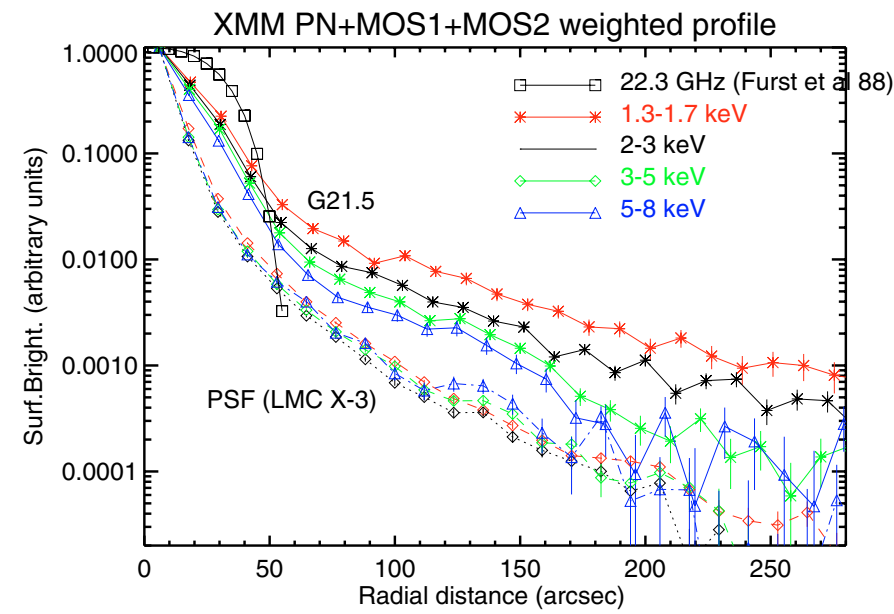

Fig. 2. X-ray surface brightness profile of G21.5-0.9 as seen by XMM-Newton. For comparison, we also plot the profile for a bright point source (LMC X-3). Moreover, we plot the profile of the source as observed by Fürst et al. (1988) at $22.3 \mathrm{GHz}$.

\section{Spectral analysis}

\subsection{The halo}

We have performed a spectral analysis of the halo using both Chandra and XMM-Newton data. We have generated single PN, MOS and ACIS-S3 spectra. The effective areas have been averaged, while we have used the standard MOS response matrix for the epoch of the observations and the on PN matrix. We have defined annulus extraction regions, namely region $1\left(0^{\prime \prime}-12^{\prime \prime}\right), 2\left(12^{\prime \prime}-24^{\prime \prime}\right), 3\left(24^{\prime \prime}-36^{\prime \prime}\right)$ and $4\left(36^{\prime \prime}-51^{\prime \prime}\right)$ for the plerion; 5 (51"-94", inner halo), 6 (94"'-152", middle halo), and 7 (152"-280", outer halo) for the halo. Regions containing the North Spur and other filamentary structure have been removed. We used a power-law emission model to fit the spectra. The background has been chosen in an annulus between 302 and 330 arcsec, excluding out-of-time events for PN. The results of spectral fits are shown in Fig. 3.

The data in region 1-7 are nicely fitted with a power-law model, with $\chi^{2} /$ d.o.f. $=365 / 390,739 / 395,338 / 361,338 / 361$, $262 / 253,242 / 205,181 / 190,258 / 261$, respectively. The derived spectral slope shows a steepening in the plerion regions 1-4 which was already measured by Warwick et al. (2001) and Safi-Harb et al. (2001). However, in the halo regions 5-7, no significant spectral steepening is observed and all these regions are consistent with a photon index value of $\sim 2.3$. It is noteworthy that the derived value of the absorbing column density is not constant in all the regions, as would be expected. The absorption is maximum in the central core and decreases monotonically towards the exterior parts. The difference in $N_{\mathrm{H}}$ between the center and the halo periphery is $\Delta N_{\mathrm{H}} \sim 10^{22} \mathrm{~cm}^{-2}$, and there is moderate evidence for flattening of the $N_{\mathrm{H}}$ decreasing trend in the halo regions. Such a high difference between the core and the halo can hardly be intrinsic, corresponding to a line of sight of $3 \mathrm{pc}$ of absorbing material at 1000 atom $\mathrm{cm}^{-3}$.

Moreover, we have also selected a region to study in detail: the bright limb of the halo between $115^{\prime \prime}$ and $138^{\prime \prime}(\mathrm{PA}=$ $\left.199^{\circ}-318^{\circ}\right)$. Spectral fits in these regions were performed to 

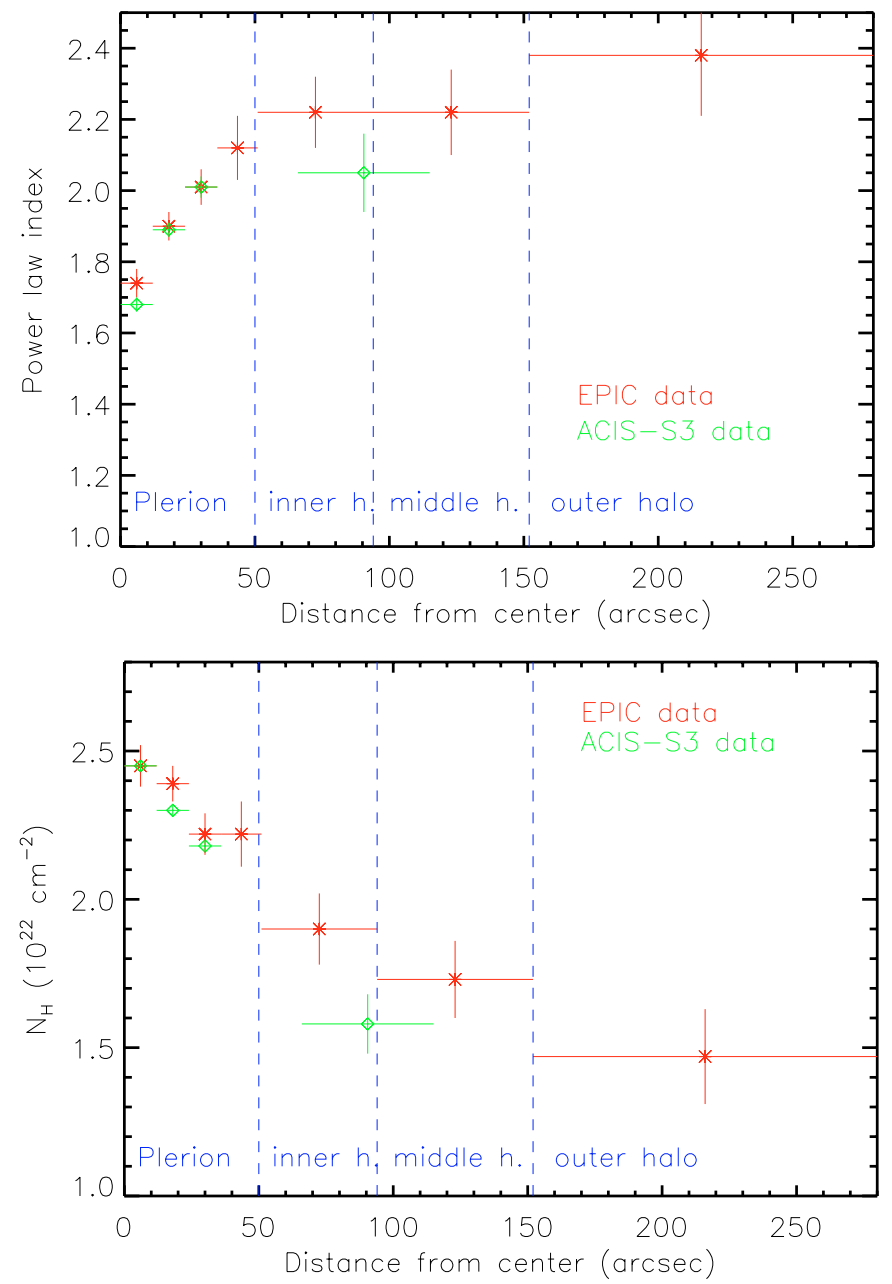

Fig. 3. Results of spectral fitting of G21.5-0.9 plerion and halo data with a power-law model modified by interstellar absorption. We show the best-fit values and $90 \%$ confidence level uncertainties for the absorbing column density and the photon spectral index.

test the presence of an additional thermal emission due to ISM (interstellar medium) heated by the forward shock, using a combination of power-law and the MEKAL model in XSPEC v11.1 (Mewe et al. 1985) with standard abundances. We have also tried the model "SRCUT" of Reynolds (1998) which represents the emission of electrons accelerated at the strong shock of a SNR shell. The latter model was used to test if the X-ray halo may be a non-thermal shell like SN1006 (Dyer et al. 2004), G347.3-0.5 (e.g. Uchiyama et al. 2003), RX J0852.0-4622 (e.g. Slane et al. 2001), and other young SNRs. The thermal component is not detected in the Chandra ACIS-S3 spectrum, and we derived an upper limit of $10^{10} \mathrm{~cm}^{-5}$ to the emission measure of $1-10 \mathrm{keV}$ plasma, corresponding to an upper limit of $0.65 \mathrm{~cm}^{-3}$ for the post-shock density and an emitting mass $<0.045 M_{\odot}{ }^{1}$. In the XMM-Newton EPIC spectrum there is a marginal detection of an excess below $1.2 \mathrm{keV}$ which requires a thermal component with $k T=0.2-0.7$. Since

\footnotetext{
${ }^{1}$ Here and in the following we assume a distance of $5 \mathrm{kpc}$ (Davelaar et al. 1986; Bock et al. 2001). The density and the mass scale as $d^{-1 / 2}$ and $d^{5 / 2}$, respectively. We have also assumed the swept-up mass is located in a thin shell.
}

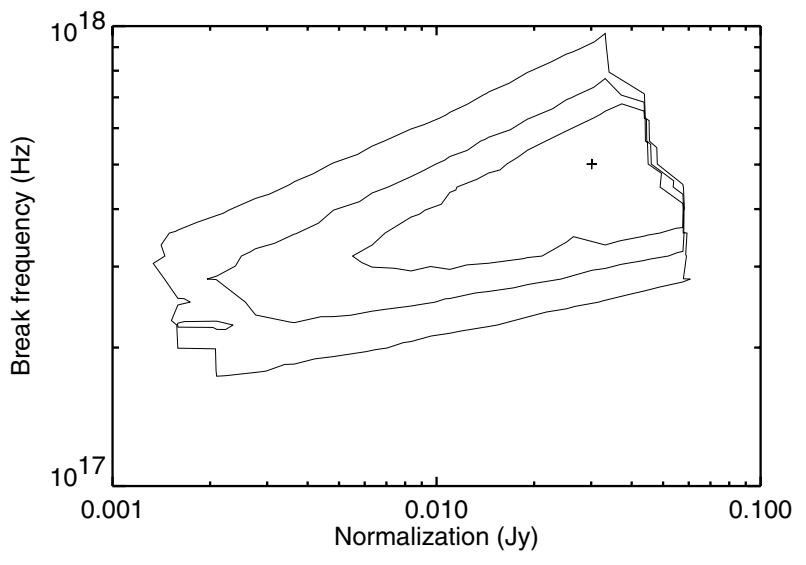

Fig. 4. 68\%, 90\% and 99\% confidence level $\chi^{2}$ contours on the spectral break vs. radio flux parameters of the SRCUT model obtained in a fit of the X-ray spectrum of the bright limb in Fig. 1.

this is not confirmed in the Chandra spectrum of the same region, and since the thermal component does not fit the spectrum in the $0.5-0.8 \mathrm{keV}$ band, we will not discuss it.

As for the fit to the "SRCUT" model, we do not know the radio flux at $1 \mathrm{GHz}$ and slope of the radio spectrum $(\alpha$, the energy index), since the halo has not been detected yet in radio (for a discussion on the parameters of the SRCUT model see Dyer et al. 2004). Therefore, we constrained $\alpha$ in the range $0.3-0.6$, which is typically observed in other nonthermal shells, and we left the radio flux and spectral break location free to vary. In this way, we may see if the extrapolation of the X-ray spectrum back to the radio regime according to the acceleration model is consistent with the upper limit of Slane et al. (2000). The fits of SRCUT model to the bright limb are as good as the power-law fits, and indicate that the upper-limit, after rescaling for the different source regions (the upper-limit is $0.08 \mathrm{Jy}$ in the rim region), is $\sim 2-3$ times above the expected radio fluxes derived with the spectral fits (see e.g. Fig. 4). The value obtained for the location of the spectral break is $2-9 \times 10^{17} \mathrm{~Hz}$, corresponding to a maximum energy of accelerated particles of $30-85(B / 10 \mu \mathrm{G}) \mathrm{TeV}$, similar to what is found in other non-thermal SNR shells.

\subsection{The North Spur}

The spectrum of the North Spur was already studied by Bocchino (2005), who reported the presence of an additional thermal component. In this work, we test if the additional thermal component is affected by Non-Equilibrium Ionization (NEI), which is expected to be present in the spectra of ejecta, circumstellar and interstellar material in the SNR. With this aim, we fitted the North Spur ACIS and EPIC data simultaneously with a combination of the power-law model, the VMEKAL model of the X-ray emission of an optically thin plasma in ionization equilibrium (Mewe et al. 1985), and the constant temperature and single ionization time NEI emission model of Borkowski et al. (2001). When fitting the powerlaw+vmekal and the powerlaw+VNEI combination, we fixed the interstellar absorption value to $N_{\mathrm{H}}=2.15 \times 10^{22} \mathrm{~cm}^{-2}$, which is similar 
Table 2. Results obtained by a ACIS-EPIC joint spectral fit of the North-Spur X-ray emission. We fixed the interstellar absorption at $N_{\mathrm{H}}=$ $2.15 \times 10^{22} \mathrm{~cm}^{-2}$.

\begin{tabular}{lcccccc}
\hline \hline $\begin{array}{l}\text { Model } \\
10^{22} \mathrm{~cm}^{-2}\end{array}$ & $\begin{array}{c}\gamma \\
\text { photon index }\end{array}$ & $\begin{array}{c}\text { Norm } \\
\mathrm{ph} \mathrm{cm}^{-2} \mathrm{~s}^{-1} \mathrm{keV}^{-1}\end{array}$ & $\begin{array}{c}k T \\
\mathrm{keV}\end{array}$ & $\begin{array}{c}\tau \\
\mathrm{cm}^{-3} \mathrm{~s}\end{array}$ & $\begin{array}{c}\text { thermal flux } \\
\mathrm{erg} \mathrm{cm}^{b} \mathrm{~s}^{-1}\end{array}$ & $\chi^{2} /$ d.o.f. \\
\hline PL & $2.45 \pm 0.05$ & $2.7 \pm 0.2 \times 10^{-4}$ & - & - & - & $876.0 / 589$ \\
PL+VMEKAL & $2.18 \pm 0.04$ & $2.0 \pm 0.2 \times 10^{-4}$ & $0.13 \pm 0.06$ & - & $2.3 \times 10^{-11}$ & $632.8 / 584$ \\
PL+VNEI (M1) & $2.15 \pm 0.04$ & $1.8 \pm 0.2 \times 10^{-4}$ & $0.17(0.15-0.21)$ & $7 \times 10^{11}$ & $1.5 \times 10^{-11}$ & $626.7 / 583$ \\
PL+VNEI (M2) & $2.15 \pm 0.04$ & $1.9 \pm 0.2 \times 10^{-4}$ & $0.30(0.20-0.37)$ & $1 \times 10^{10}$ & $1.7 \times 10^{-11}$ & $628.8 / 583$ \\
\hline
\end{tabular}

${ }^{a} \mathrm{PL}=$ power-law.

${ }^{b}$ Unabsorbed flux in the $0.5-2.0 \mathrm{keV}$ band due to the thermal component only.

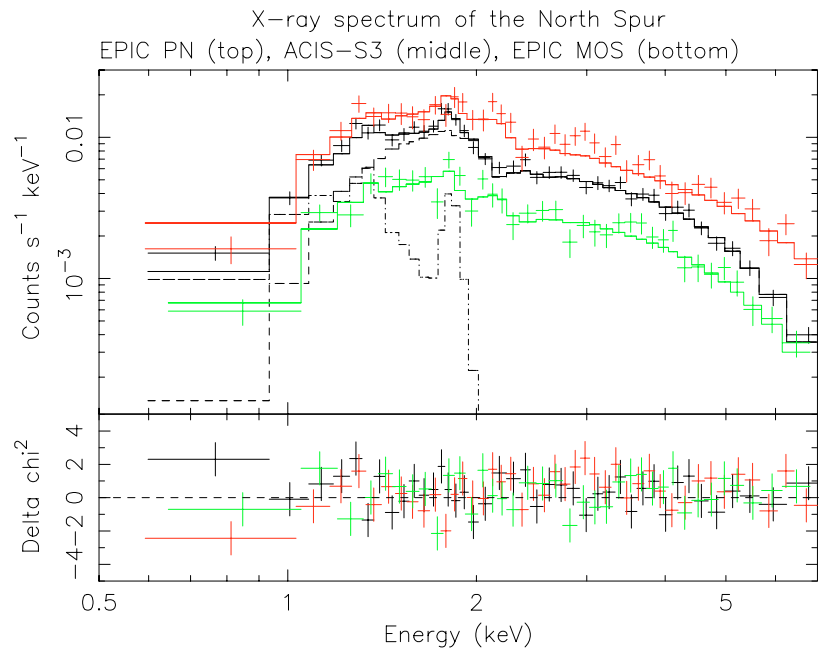

Fig. 5. Chandra ACIS-S3 and XMM-Newton PN and MOS spectra of the North-Spur, along with the best-fit NEI model. We also show the contribution of thermal and non-thermal component to the ACIS spectrum (dot-dashed and dashed lines, respectively).

to the best-fit value obtained when this parameter is left free to vary. The results are summarized in Table 2, while the ACIS and EPIC spectra along with their NEI best-fit model are shown in Fig. 5. The X-ray spectrum (especially the ACIS-S3 spectrum) shows signs of the presence of two bright emission lines, namely $\mathrm{Mg}$ XI at $1.34 \mathrm{keV}$ and Si XIII at $1.86 \mathrm{keV}$ (Fig. 5).

Unfortunately, the combination of the power-law and NEI models gives two local minima, one which represents the equilibrium situation already found with MEKAL fit $\left(\chi^{2} /\right.$ d.o.f. $=626.7 / 583$, hereafter M1 $)$, and one which represents a plasma strongly affected by NEI conditions $\left(\chi^{2} /\right.$ d.o.f. $=$ $628.8 / 583$, hereafter M2). Both results are presented in Table 2 and displayed in Fig. 6.

The minimum M1, which has a slightly lower $\chi^{2}$ value then M2, yields parameters similar to the ones found by Bocchino (2005). On the other hand, the minimum M2 gives an ionization time between $7 \times 10^{9}$ and $2 \times 10^{10} \mathrm{~cm}^{-3} \mathrm{~s}$ and a temperature in the range $0.2-0.4 \mathrm{keV}$ (at the $90 \%$ confidence level), a factor of two higher than M1 (Fig. 6, top panel).

The NEI results at minimum M2 lead to an emitting plasma density of 5(3-8) $\mathrm{cm}^{-3}$ and mass of $0.23(0.17-0.37) M_{\odot}$, if the line of sight extension of the emitting plasma is equal to the chord intersecting the sphere of the SNR shell $(\sim 6.5 \mathrm{pc})$
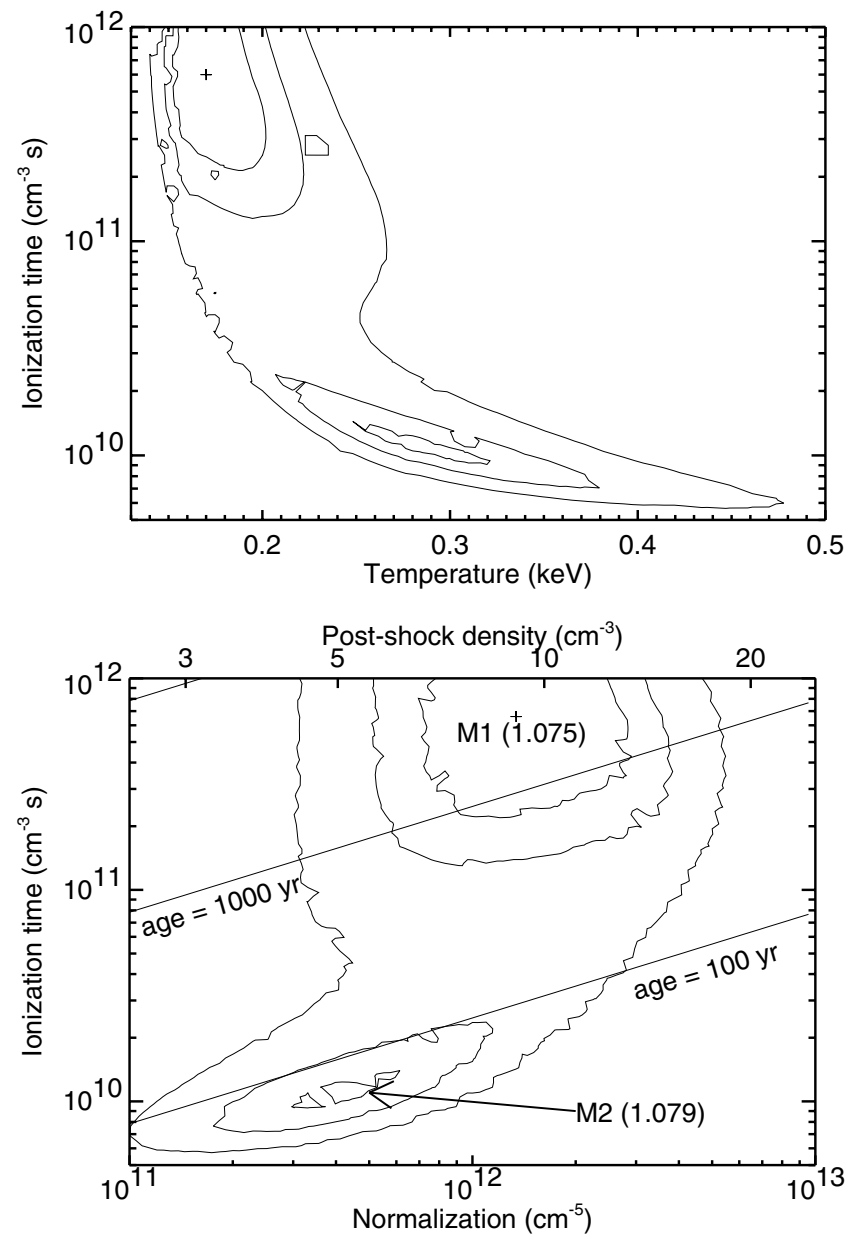

Fig. 6. Ranges of $k T$ and ionization time (top panel), emission measure and normalization time (bottom panel) allowed by the fit to North Spur data with a combination of power-law and NEI emission model. In the bottom panel, we also show the range of post-shock density and the range of ages which are compatible with the EM and $\tau$ values derived from the fit.

At the minimum M1 the corresponding values are $\sim 10 \mathrm{~cm}^{-3}$ and $\sim 0.5 M_{\odot}$, respectively. It is noteworthy that the derived age of the North Spur compatible with the M2 minimum is $\sim 100 \mathrm{yr}$, which is significantly lower than the corresponding age at M1 ( $\approx 1000$ yr, Fig. 6, bottom panel).

The metal abundances of $\mathrm{Mg}$ and $\mathrm{Si}$, as measured by the minimum M1 are 0.6-3 times the solar value for $\mathrm{Mg}$ and 2-20 


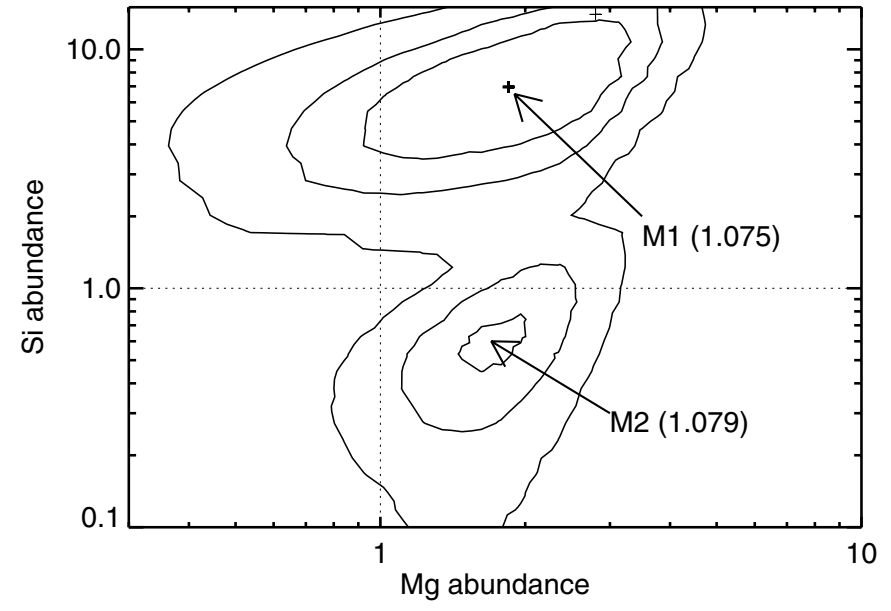

Fig. 7. $\mathrm{Mg}$ and $\mathrm{Si}$ abundances obtained by fitting with a powerlaw and VNEI model. The locations of the two minima (M1 and M2) are marked by arrows, and the $\chi^{2}$ is reported.

for $\mathrm{Si}$, but for M2 the abundances are consistent with the solar values for both elements (Fig. 7). The measured thermal flux corresponds to a luminosity of $\sim 4 \times 10^{34} \mathrm{erg} \mathrm{s}^{-1}$ in the $0.5-2.0 \mathrm{keV}$ band. This luminosity is a bit high compared to an ejecta knot of Cas A, for which from the results published by Laming \& Hwang (2003) we have computed $L_{\max } \sim$ $10^{34} \mathrm{erg} \mathrm{s}^{-1}$. However, our measurement of the unabsorbed thermal flux is affected by large uncertainty, which can increase or decrease the luminosity by a factor of 10 . The total absorbed flux of the North Spur in the 0.5-2.0 keV band is $3.2 \times 10^{-13} \mathrm{erg} \mathrm{cm}^{-2} \mathrm{~s}^{-1}$, of which $\sim 25 \%$ is due to the thermal component.

\section{A dust scattering model for the "pure" halo}

In this section, we investigate whether the diffuse emission of the G21.5-0.9 X-ray halo may be due to dust scattering of $\mathrm{X}$-ray photons from the plerion. There are reasons to suspect that dust scattering contributes appreciably to the G21.5-0.9 $\mathrm{X}$-ray profile. One is the large absorption column density toward this source. Dust scattering X-ray halos are typically found around other heavily absorbed sources (see e.g. Predehl \& Schmitt 1995; Smith \& Dwek 1998; Nagase et al. 2001; Vaughan et al. 2004). Predehl \& Schmitt (1995) have shown that there is a good correlation between the optical depth for dust scattering and the absorption column density. Using their empirical formula, for G21.5-0.9 one expects $\tau_{1 \mathrm{keV}} \simeq 1$ (where $\tau_{1 \mathrm{keV}}$ is the scattering optical depth a $1 \mathrm{keV}$ ). For the sake of illustration, $\tau_{1 \mathrm{keV}}=1$ implies a halo fractional flux of about $50 \%, 20 \%, 7 \%$ and $3 \%$, respectively for the energy bands 1.3-1.7 keV, 2-3 keV, 3-5 keV and 5-8 keV (these will be the reference energy bands, used in our analysis). However, there is a large scatter about that correlation, so that it cannot be taken as a safe method to evaluate $\tau_{1 \mathrm{keV}}$ in individual sources.

Another effect that could be also ascribed to dust scattering is the systematic decrease of the absorption column density with the distance from the source center (see Fig. 3), as derived from X-ray spectral analysis. If it was a true column density variation, it would require an improbable distribution of the foreground matter. Instead, a lower measured absorption in the outer regions can be justified as an artifact of the spectral analysis, in the presence of dust scattering, due to the fact that hard X-rays are scattered at lower angles than soft X-rays.

We present here an analysis along the lines of that by Bandiera \& Bocchino (2004), but carried out with much better accuracy. First, we start from data of better quality, because they are based on a longer integration time, and with more careful selection of the directions along which the contamination from the North Spur and the shell is the lowest. Then, we have corrected for some small bias in the profile normalization, which was affecting the analysis of Bandiera \& Bocchino (2004). Finally, we have performed a much better analysis on the parameter space.

In short, the procedure is as follows. We assume that the dust halo is negligible in the $5-8 \mathrm{keV}$ band (we have already seen that, for $\tau_{1 \mathrm{keV}}=1$, the halo fractional flux in this band is only $3 \%$ ). Therefore, the radial profile in the $5-8 \mathrm{keV}$ band is well approximated by the convolution of the intrinsic source profile with the instrumental Point Spread Function (PSF). Let us assume that the PSF is energy independent (this is a good approximation, for EPIC onboard XMM-Newton) and also that the source intrinsic radial profile does not change much with the energy (this assumption will be verified "a posteriori"). In this case, the 5-8 keV radial profile should reproduce rather well profiles in other bands, except for the different flux normalizations and for the further scattering component, which becomes more prominent in softer energy bands. For a given model of the scattering halo, the scattering component is thus obtained as its convolution with the PSF. Finally, the sum of the intrinsic and scattering components must be compared with the profile measured in a given energy band (we shall use $2-3 \mathrm{keV}$ ). A least $\chi^{2}$ analysis allows one to constrain the parameters of the halo model. This is a rather time-consuming procedure, because for each set of parameters we are required to compute one convolution of 2-dimensional maps.

It is well known that, as long as the Rayleigh-Gans approximation is valid, the scattering optical depth scales as $E^{-2}$ (where $E$ is the photon energy), while the angular size of the halo scales with $E^{-1}$ (see e.g. Predehl 1998, for a discussion of these scaling laws). For the angular size, a formula similar to that of classical diffraction applies, namely scattering angles are of the order of the ratio between photon wavelength and dust grain size. Using this scaling law, we can then simulate profiles for the other two energy ranges, 1.3-1.7 keV and 3-5 keV, and compare them with observations.

However, it should be clear that even in this sophisticated analysis we can only check for self-consistency of a scattering halo model, while we cannot prove for sure the dust-scattering nature of an X-ray halo. In principle, both spatial and spectral properties of a source may conspire to mimic shape and scaling of a dust-scattering halo. However, the required conditions would be highly unlikely, and this is the reason why we finally conclude that the dust-scattering halo hypothesis is confirmed to a high confidence level.

The relevant parameters to model a dust-scattering halo are the following: the optical depth for scattering (at the reference 
energy, say $\tau_{1 \mathrm{kev}}$ ); the dust distribution along the line of sight (here we shall use a uniform distribution); the size distribution of grains, usually approximated by a power-law with an upper cutoff ( $\propto a^{-q}$ for $a<a_{\max }$; typical values are $a_{\max }=0.17 \mu \mathrm{m}$ and $q=3.9$, Predehl \& Schmitt 1995).

For the scattering halo, we use a simplified, analytic model, that will be described in a forthcoming paper, together with details of the procedure of halo modeling and subtraction in individual sources. We outline here only some basic features of this model and of halo models in general (under RayleighGans, and single-scattering approximations).

If the space distribution of dust is uniform along the line of sight $^{2}$ then the halo profile is $\propto \theta^{-1}$, for $\theta \ll \theta_{\text {scal }}$, where

$\theta_{\text {scal }}(x) \simeq \frac{3200 \operatorname{arcsec}}{(E / 1 \mathrm{keV})\left(a_{\max } / 0.17 \mu \mathrm{m}\right)}$

The range of radial distances over which we see the halo in G21.5-0.9 is about 100-300 arcsec. Therefore, from Eq. (1) it follows that we are in the $\theta \ll \theta_{\text {scal }}$ regime. A consequence of this (as we have found by analyzing numerically the parameter space) is that the best fit halo does not constrain at all the value of $q$ : then, we have assumed for this parameter the average value 3.9, as given by Predehl \& Schmitt (1995).

For the minimum $\chi^{2}$ fit, we have used errors proportional to the measured values, because the radial profile within $\sim 100$ arcsec is much more uncertain than one would infer from the (very small) statistical error (here the main uncertainty derives from the fact that the intrinsic source profile slightly changes with energy). The best-fit we got is shown in Fig. 8, along with the derived $\tau$ (in the $2-3 \mathrm{keV}$ band) and $\theta_{\text {scal }}$. We thus estimate $\tau_{1 \mathrm{keV}}=5.68 \tau_{2-3 \mathrm{keV}} \simeq 0.80$, with a $1 \sigma$ uncertainty of about $20 \%$.

In Fig. 9 we show an image of G21.5-0.9 in the band 2-8 keV with the best-fit dust scattering model profile subtracted. The emission which remains after the halo subtraction is the residual flux from the plerion (which is not expected to be properly modeled by a circularly symmetric model), the North Spur, its filaments and the shell. In particular, the shell is clearly visible from $\mathrm{PA}=180^{\circ}$ to $300^{\circ}$, while it is not visible at $\mathrm{PA}=90^{\circ}-180^{\circ}$.

In a separate paper, we will report more details on the dust scattering model and the fit to the G21.5-0.9 X-ray halo, including a thin slab dust distribution and a discussion of the dust properties we found, in the framework of general dust properties as derived from studies of X-ray halos in other sources.

\section{A self-consistent model for the X-ray halo and its features}

\subsection{Establishing the evolutionary stage of G21.5-0.9}

As we have seen, the bright limb in Figs. 1 and 9 suggests that we have detected the forward shock of G21.5-0.9 expanding into the environment of the SNR. If this is the case, we

\footnotetext{
2 We have tried to relax this hypothesis by considering a thin slab of scattering dust at a given distance. The chi-square analysis shows that the "uniform" case has to be preferred. More elaborate and complex scenarios are not justified by the present data quality.
}
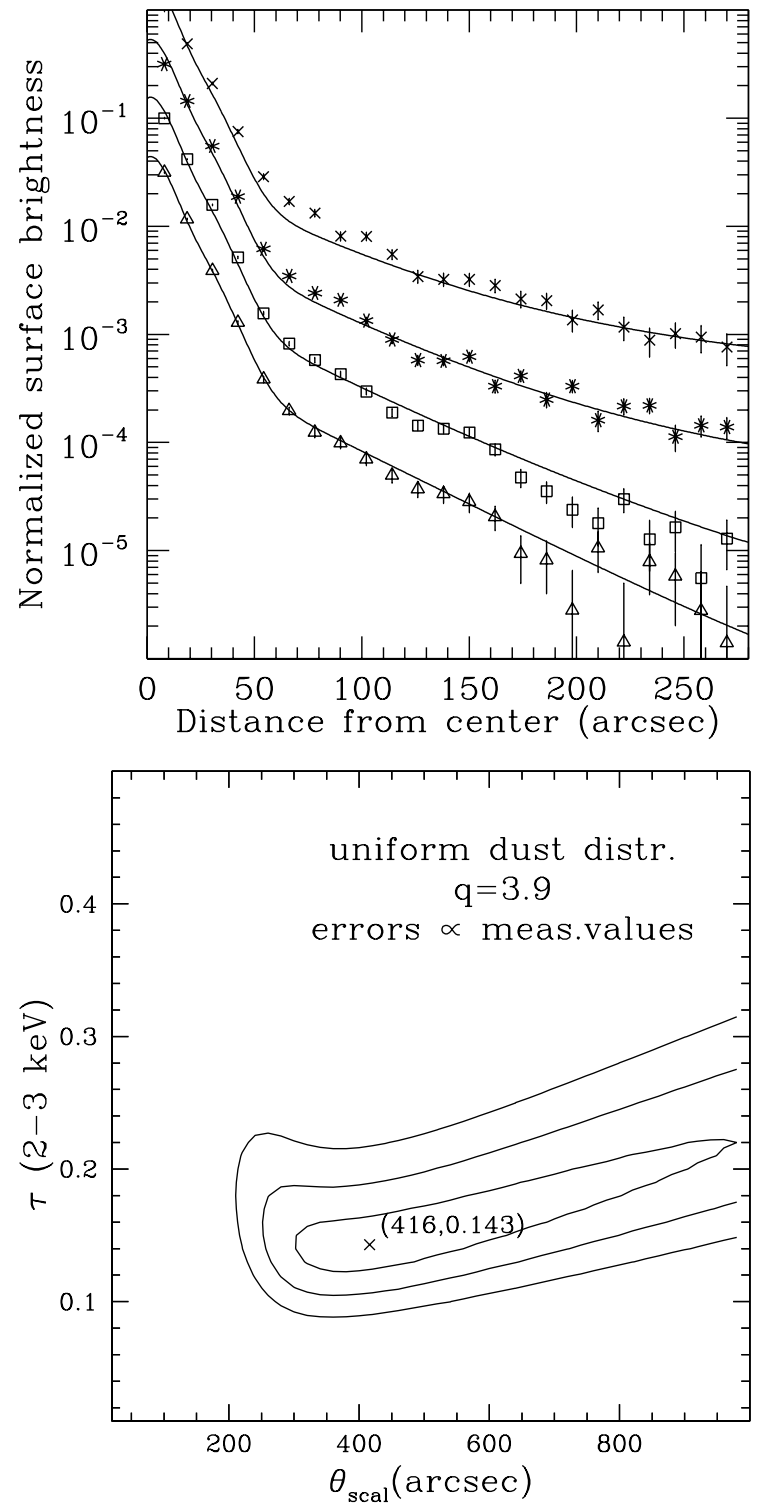

Fig. 8. Top panel: G21.5-0.9 X-ray profiles with best-fit dust scattering model (crosses $1.3-1.7 \mathrm{keV}$, asterisks $2-3 \mathrm{keV}$, diamonds $3-5 \mathrm{keV}$, triangles $5-8 \mathrm{keV}$ ). The derived dust parameters and their uncertainties are shown in the bottom panel.

may use evolutionary models of young SNR to infer the age of G21.5-0.9. First, we argue that the remnant is not yet in the Sedov-Taylor (ST) phase, because the swept-up mass by the shock front in the bright limb, as derived in Sect. 4, is very low $\left(<0.045 M_{\odot}\right)$. Even taking into account the mass in the North Spur and the other filaments not analyzed, the total swept-up mass in the halo is less than $0.5 M_{\odot}$.

Chevalier (2005) has discussed the interaction of a young core-collapse SNR with its environment, taking into account different supernova types, namely SN1987A-like class, IIP, $\mathrm{IIL} / \mathrm{b}$, and Ib/Ic. Because of the low mass observed in the halo, we may safely discard a dense environment for G21.5-0.9 like the RSG wind usually found for type IIL/b. In fact, it can be shown that for an observed radius of 3.3 pc (Sect. 3), the remnant of a type IIL/b with a dense RSG wind should have 


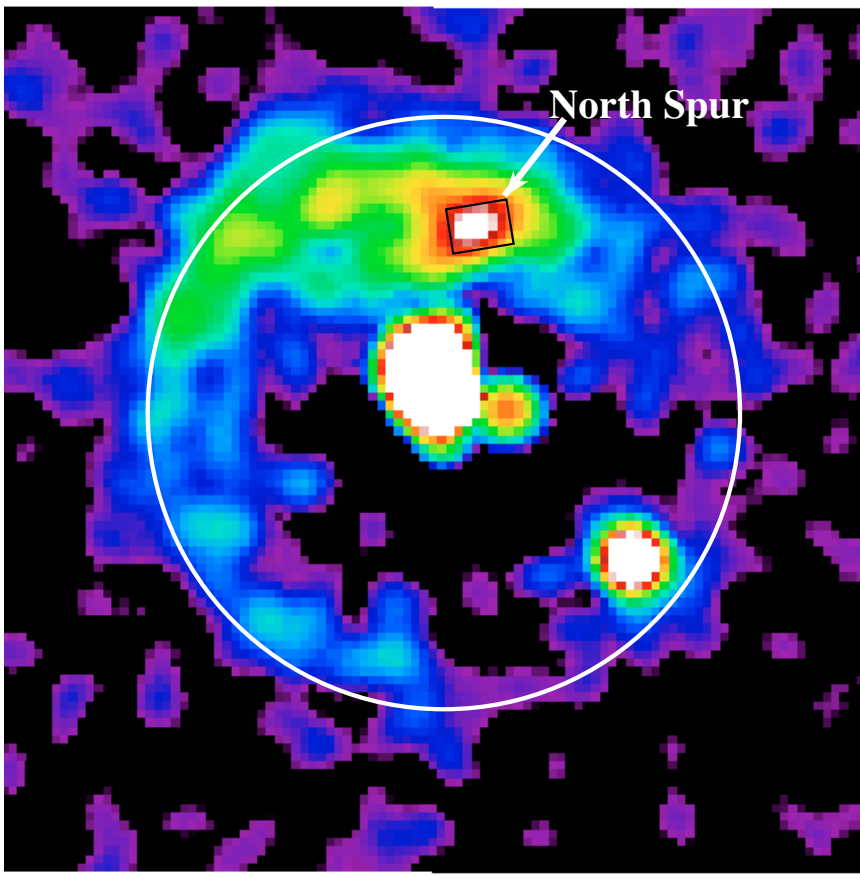

Fig. 9. Image of G21.5-0.9 in the $2-8 \mathrm{keV}$ band with dust scattering contribution subtracted.

swept-up most of the total ejecta mass and a few solar masses of circumstellar medium.

Type IIP and Ib/Ic are interesting possibilities. For type IIP, Chevalier (2005) notes that the low mass loss during the RSG phase would result in a small region $(r<1 \mathrm{pc})$ of dense wind surrounded by a more diffuse extended bubble created during the main sequence phase. The interaction with the resulting double layer wind structure has not been modeled in detail but swept-up masses are of the order of $0.1 M_{\odot}$, so in agreement with observations. For type $\mathrm{Ib} / \mathrm{Ic}$, it is expected that they undergo a Wolf-Rayet star phase with high mass-loss and fast wind which sweeps the earlier RSG bubble in a shell at several parsecs from the center. The evolution of the SNR in this medium may also be complicated and requires numerical simulation (Dwarkadas 2001). However, if we assume that the star has been a WR object long enough to produce an extended wind component, we may use the interaction model developed by Chevalier (2005) for the circumstellar interaction of an RSG wind, but with the parameters appropriate for a WR wind $\left(E_{51}=1, M_{\mathrm{ej}}=4 M_{\odot}, \dot{M}=3 \times 10^{5} M_{\odot} \mathrm{yr}^{-1}\right.$, wind velocity $v_{\mathrm{w}}=1000 \mathrm{~km} \mathrm{~s}^{-1}, D=\dot{M} / 4 \pi v_{\mathrm{w}}=1.5 \times 10^{12} \mathrm{~g} \mathrm{~cm}^{-1}$ ). The model assumes that the progenitor star had a radiative envelope and treats the interaction in the thin shell approximation. Although the progenitor star of G21.5-0.9 may not have had a radiative envelope, the steep outer power law with a relatively flat central density distribution is probably a reasonable approximation to the density profile. We assume that the interaction shell is still in the outer steep power law part of the supernova density profile; this assumption can be verified for the parameters we find for G21.5-0.9. In this case, we derive a CSM swept-up mass of $0.1 M_{\odot}$, an ejecta swept-up mass of $0.2 M_{\odot}$, which are again roughly in agreement with observed

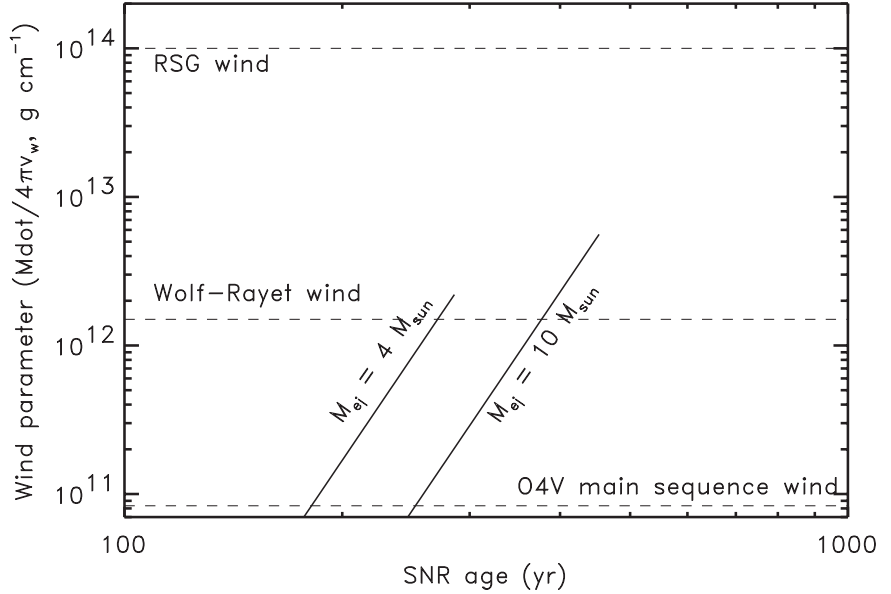

Fig. 10. The CSM interaction model of Chevalier (2005) adapted to the G21.5-0.9 case. The two segments are the loci of allowed wind parameter and SNR age values calculated for 4 and $10 M_{\odot}$ ejecta masses and an upper-limit of $0.1 M_{\odot}$ for the CSM swept-up in a shell, in agreement with the results obtained in Sect. 4.1 for the bright halo. The wind parameters for three kinds of stars are also shown, a RSG wind (which turns out to be incompatible with the data for any reasonable value of the system parameters), a WR star wind (one of the viable interpretations for the G21.5-0.9 progenitor), and a O4V normal star, shown only as a reference for the minimum value the wind parameter may have.

mass in the North Spur and the upper-limit of the shell. The derived shell velocity is $\sim 7000 \mathrm{~km} \mathrm{~s}^{-1}$, and the age is $\sim 290 \mathrm{yr}$. For any reasonable set of wind parameters, the upper-limit on the swept-up mass derived in Sect. 4.1 implies an age lower then $\sim 450$ yr. This is shown in Fig. 10 where allowed SNR ages are plotted for different wind parameters and ejecta masses according to the self-similar solution of the CSM interaction model of Chevalier (2005), adapted to the observed size of G21.5-0.9, and considering only a swept-up CSM mass below $0.1 M_{\odot}$, in agreement with the observations.

Therefore, the spectral results for the shell and the North Spur, when combined with reasonable scenarios of the SN environment, seem to indicate in any case an early evolutionary stage for the SNR and a progenitor without extended dense winds. We may now see if this is also compatible with the size of PWN and some appropriate model for PWN-SNR interaction. Several authors (Reynolds \& Chevalier 1984; Chevalier \& Fransson 1992; van der Swaluw et al. 2001) give analytical expressions which relate the radius of the PWN shock and the SNR blastwave. We have applied these equations to the case of G21.5-0.9 using a SNR shock radius of 3.3 parsec and a PWN shock radius of 1.2 parsec, under the assumption of a uniform ejecta density and constant spin-down luminosity. We typically get $E_{\mathrm{pwn}}=L_{0} t \sim 10^{49} \mathrm{erg}$ and an age of $\sim 500 \mathrm{yr}$ from these models. While the age is in agreement with the CSM interaction model, the corresponding (constant) spin-down luminosity is very high especially considering that the $\mathrm{X}$-ray luminosity is considerably less than that observed from the brightest PWNe, so this seems to suggest that significant spin-down has occurred at the early stage of the PWN evolution. 
Table 3. A comparison of G21.5-0.9 linear size and equipartition energy with a set of similar plerion with known age. From the comparison, one would guess a G21.5-0.9 age in the range 800-1600 yr.

\begin{tabular}{lccccc}
\hline \hline SNR & $0540-69$ & Kes75 & G11.2 & G54.1 & G21.5-0.9 \\
\hline$R(\mathrm{pc})$ & 0.9 & 1.4 & 0.9 & 1.2 & 1.2 \\
Age & 800 & 1000 & 1600 & 1500 & $?$ \\
$E_{\min }$ & $5 \times 10^{47}$ & $10^{48}$ & $3 \times 10^{46}$ & $8 \times 10^{46}$ & $4 \times 10^{47}$ \\
\hline
\end{tabular}

Chevalier (2005) has developed a model of PWN interaction with SN ejecta, considering power-law density profile for the ejecta and the pulsar luminosity decay. In the framework of his model, it is convenient to compare the PWN internal energy with the pulsar luminosity and the shell kinetic energy. The equipartition energy of the nebula can be found in the following way

$E_{\min }=3.2 \times 10^{47}\left(\frac{L_{\mathrm{b}}}{3.6 \mathrm{Jy}}\right)^{\frac{4}{5}}\left(\frac{R_{\mathrm{PWN}}}{1.2 \mathrm{pc}}\right)^{\frac{9}{7}}\left(\frac{v_{\mathrm{b}}}{540 \mathrm{GHz}}\right)^{\frac{2}{7}}$

where $L_{\mathrm{b}}$ is the spectral luminosity at the break frequency $v_{\mathrm{b}}$ (values for G21.5-0.9 are from Bandiera et al. 2001), the particle energy indexes before and after the break are $p_{1}=1$ and $p_{2}=3$, respectively (adapted from Chevalier 2005). The nebula internal energy is usually within a factor of a few of $E_{\mathrm{min}}$, say $E_{\mathrm{int}}=10^{48} \mathrm{erg}$. Safi-Harb et al. (2001) found $\dot{E}=3 \times 10^{37} \mathrm{erg} \mathrm{s}^{-1}$, based on the $\dot{E}-L_{\mathrm{X}}$ relation, implying $\dot{E} t=4.7 \times 10^{47} t_{500} \mathrm{erg}$, where $t_{500}$ is the age in units of $500 \mathrm{yr}$. The kinetic energy in the PWN is $\sim 10^{49} \mathrm{erg}$, which is determined by the supernova model and the age. This set of energies is consistent with the model if the ratio of the age and the initial spin-down timescale $t / \tau$ is in the range $1-10$ (see Fig. 1 in Chevalier 2005), thus suggesting that significant spindown can occur at the early stage of the PWN evolution.

Finally, we should note that the comparison of the equipartition energy and linear dimension of G21.5-0.9 with other PWNe, summarized in Table 3, seems to suggest an age of 800-1600 yr, older then what we found on the basis of the circumstellar interaction model. One way of having the PWN expand more rapidly is to have a lower density supernova, but this generally requires a lower mass or higher energy, which also reduce the age from the circumstellar interaction model.

\subsection{On the nature of "North Spur"}

In this section, we review a physical interpretation for the North Spur which is compatible with the observational results. Figure 1 shows that the spur is located at $35^{\prime \prime}(0.8 \mathrm{pc})$ outside the plerion rim, with a low surface brightness in between. This is at odds with an interpretation in terms of material swept-up by the plerion, because in that case we expect the thermal emission adjacent or inside the plerion non-thermal emission (as in, e.g., 3C 58, Bocchino et al. 2001).

Therefore, a possible valid interpretation may be that the North Spur is an ejecta clump hit by the reverse shock. In this case, the position of the forward shock $\left(R_{\mathrm{fs}}\right)$, as traced by the bright limb in the halo, and the contact discontinuity $\left(R_{\mathrm{c}}\right)$, as traced by the emission from the North Spur, can be compared with self-similar solutions of interaction of ejecta with an external medium as worked out by Chevalier (1982). For a model in which ejecta with steep power-law outer density distribution ( $n>5$, where $n$ is the power-law index) are interacting with the free stellar wind of the massive progenitor $(s=2$, where $s$ is the power-law index of stationary medium, usually mass-loss from progenitor star), the forward shock is expected to be at $\lesssim 1.3-1.4 R_{\mathrm{c}}$, unless it has already entered the flat part of ejecta density distribution. Unfortunately, because of projection effects, the exact location of the North Spur inside the remnant is not exactly known, but it is between $1.0 R_{\mathrm{c}}$ and $1.7 R_{\mathrm{c}}$, so in general agreement with expectation. As already noted in Sect. 4.2, the density, mass and luminosity estimates for the North Spur are not unusual for ejecta in young SNRs. However, the measured temperature is lower then the one expected for emitting X-ray ejecta and usually observed in other young SNRs originating from a type II SN (e.g. Cas A, Gotthelf et al. 2001), thus casting some doubt on this interpretation.

A different explanation for the North Spur which takes into account the low X-ray temperature may be the following. We have seen the a progenitor SN of type IIP is in agreement with the observationally derived masses. Chevalier (2005) show that in case of a type IIP SN, the circumstellar interaction should occur early (radius $<1 \mathrm{pc}$ ), followed by adiabatic expansion of the reverse shocked ejecta. This adiabatic expansion could give rise to the relatively cool emission which is observed. The mass is not expected to be high and it is in agreement with the observed value of $\sim 0.2 M_{\odot}$. Other implications of this scenario are a young age and near solar abundances (the envelope of the the type IIP SN), which are both in agreement with the age and abundances derived at the minimum M2 (Fig. 6), and the dynamical age of the shell derived in the previous section.

We also note that the data indicate that the North Spur has an intrinsic non-thermal tail in the spectrum that is modeled with a power-law $(\gamma=2.15$, Table 2$)$. One explanation for the non-thermal emission is synchrotron radiation from particles accelerated in a shock.

\section{Conclusions}

We presented an extensive analysis of Chandra and $X M M$-Newton X-ray data of the radio-quiet halo around the plerion G21.5-0.9. We included in our analysis all the public observations of the source available up to now. We showed that the halo morphology is composed of two components: diffuse emission and some bright knots and filaments. We confirmed the detection of X-ray thermal emission in the brightest knot, the "North Spur", already detected by Bocchino (2005), and we presented a more detailed spectral analysis of this object which included Non-Equilibrium Ionization, and which yielded a very young age (100-1000 yr) and abundances compatible with solar values. We have presented various interpretations for the origin of this knot, and we argue that it can be due to ejecta interaction with the $\mathrm{H}$ envelope of a type IIP SN.

We have also detected a bright limb in the east part of the halo, located at $3.3 \mathrm{pc}$ from the center. The limb is dominated 
by non-thermal X-ray emission, probably due to particle acceleration at the fast forward shock, while the upper limits for the post-shock density and emitting mass are $0.65 \mathrm{~cm}^{-3}$ and $0.045 M_{\odot}$ in this region.

We showed that the diffuse emission from the halo is due to dust scattering of X-rays from the plerion, and we explored which range of dust parameters is compatible with observations.

We argued that our data are inconsistent with an explanation of the halo in terms of a plerion extension, as suggested by previous works. We exclude the possibility that the system is in Sedov-Taylor stage on the grounds that the swept-up mass is very low. Moreover, by applying a model of CSM interaction of a young SNR shock wave to the bright limb data, we argue that G21.5-0.9 is in a very early evolutionary stage, with an age between 250 and $500 \mathrm{yr}$. A comparison of the plerion linear size and equipartition energy with the corresponding values of plerions with known age suggest a longer age (800-1600), while the comparison with a model of SNR-PWN interaction for young remnant allows age as low as $500 \mathrm{yr}$ and indicates that spindown has already occurred. Putting together all the estimates, it seems that a very reasonable range for the age of G21.5-0.9 is 200-1000 yr. The lack of an historical supernova associated with this object is not a surprise given the high extinction in this direction.

If our conclusions are correct, the PWN may be expanding at a few times $1000 \mathrm{~km} \mathrm{~s}^{-1}$, and a comparison of X-ray archive images at a baseline of $5 \mathrm{yr}$ may lead to the direct detection of PWN expansion. Moreover, additional X-ray observations would be required to study in more detail the thermal emission both from the forward shock (if any) and in the North Spur. Deeper radio observations of the North Spur and the bright limb which lead to detection of these objects would shed light on the nature of their non-thermal emission. The required radio sensitivity at $1 \mathrm{GHz}$ to detect the limb is $2-3$ times below the current upper-limit. As for the halo X-ray diffuse extended component, if it is indeed due to dust scattering, it should not have any radio counterpart. The detection of the pulsar would be of great value to further constrain the evolutionary stage of the system.

Note added after acceptance: In April 2005, during the referee review of this manuscript, Matheson \& Safi-Harb (2005) reported the results of a spectral analysis based on a Chandra dataset larger then the one used by us. The results of Matheson \& Safi-Harb (2005) confirm that the interstellar absorption increases toward the outer edge of the halo, with measured values consistent with our values. Moreover, a limb brightnening in the halo is also reported by them.

Acknowledgements. RAC was partially supported by NASA grant NAG5-13272. We are grateful for the stimulating atmosphere and support provided by the ISSI (International Space Science Institute, Bern) workshop on the "Physics of Supernova Remnants in the XMMNewton, Chandra and INTEGRAL Era.". E.v.d.S. was partially supported by PPARC. F.B. and R.B. were partially supported by INAF grant PRIN2003 "The impact of high-resolution X-ray data on the study of SNRs".

\section{References}

Asaoka, I., \& Koyama, K. 1990, PASJ, 42, 625

Bandiera, R., \& Bocchino, F. 2004, Adv. Space Res., 33, 398

Bandiera, R., Neri, R., \& Cesaroni, R. 2001, in Young Supernova Remnants, AIP Conf. Proc., 565, 329

Becker, R. H., \& Kundu, M. R. 1976, ApJ, 204, 427

Becker, R. H., \& Szymkowiak, A. E. 1981, ApJ, 248, L23

Biggs, J. D., \& Lyne, A. G. 1996, MNRAS, 282, 691

Bocchino, F. 2005, Adv. Space Res., 38, in press

Bocchino, F., Warwick, R. S., Marty, P., et al. 2001, A\&A, 369, 1078

Bock, D. C.-J., Wright, M. C. H., \& Dickel, J. R. 2001, ApJ, 561, L203

Borkowski, K. J., Lyerly, W. J., \& Reynolds, S. P. 2001, ApJ, 548, 820 Chevalier, R. A. 1982, ApJ, 258, 790

Chevalier, R. A. 2005, ApJ, 619, 839

Chevalier, R. A., \& Fransson, C. 1992, ApJ, 395, 540

Davelaar, J., Smith, A., \& Becker, R. H. 1986, ApJ, 300, L59

De Luca, A., \& Molendi, S. 2004, A\&A, 419, 837

Dwarkadas, V. V. 2001, J. Korean Astron. Soc., 34, 243

Dyer, K. K., Reynolds, S. P., \& Borkowski, K. J. 2004, ApJ, 600, 752

Fürst, E., Handa, T., Morita, K., et al. 1988, PASJ, 40, 347

Gondoin, P., Aschenbach, B. R., Beijersbergen, M. W., et al. 1998, in X-Ray Optics, Instruments, and Missions, ed. R. B. Hoover, \& A. B. Walker, Proc. SPIE, 3444, 278

Gotthelf, E. V., Koralesky, B., Rudnick, L., et al. 2001, ApJ, 552, L39 Jansen, F., Lumb, D., Altieri, B., et al. 2001, A\&A, 365, L1

Kassim, N. E. 1992, AJ, 103, 943

La Palombara, N., \& Mereghetti, S. 2002, A\&A, 383, 916

Laming, J. M., \& Hwang, U. 2003, ApJ, 597, 347

Matheson, H., \& Safi-Harb, S. 2005, Adv. Space Res., 35, 1099

Mewe, R., Gronenschild, E. H. B. M., \& van den Oord, G. H. J. 1985, A\&AS, 62, 197

Nagase, F., Dotani, T., Endo, T., et al. 2001, X-ray Astronomy: Stellar Endpoints, AGN, and the Diffuse X-ray Background, AIP Conf. Proc., 599, 794

Predehl, P. 1998, Ap\&SS, 258, 89

Predehl, P., \& Schmitt, J. H. M. M. 1995, A\&A, 293, 889

Reynolds, S. P. 1998, ApJ, 493, 375

Reynolds, S. P., \& Chevalier, R. A. 1984, ApJ, 278, 630

Safi-Harb, S., Harrus, I. M., Petre, R., et al. 2001, ApJ, 561, 308

Slane, P., Chen, Y., Schulz, N. S., et al. 2000, ApJ, 533, L29

Slane, P., Hughes, J. P., Edgar, R. J., et al. 2001, ApJ, 548, 814

Smith, R. K., \& Dwek, E. 1998, ApJ, 503, 831

Strüder, L., Briel, U., Dennerl, K., et al. 2001, A\&A, 365, L18

Turner, M. J. L., Abbey, A., Arnaud, M., et al. 2001, A\&A, 365, L27

Uchiyama, Y., Aharonian, F. A., \& Takahashi, T. 2003, A\&A, 400, 567

van der Swaluw, E., Achterberg, A., Gallant, Y. A., \& Tóth, G. 2001, A\&A, 380, 309

Vaughan, S., Willingale, R., O’Brien, P. T., et al. 2004, ApJ, 603, L5

Warwick, R. S., Bernard, J., Bocchino, F., et al. 2001, A\&A, 365, L248

Weisskopf, M. C., O'dell, S. L., \& van Speybroeck, L. P. 1996, in Multilayer and Grazing Incidence X-Ray/EUV Optics III, ed. R. B. Hoover, \& A. B. Walker, Proc. SPIE, 2805, 2

Woltjer, L., Salvati, M., Pacini, F., \& Bandiera, R. 1997, A\&A, 325, 295 